\title{
Tenue d'ancrages LMS sur fonds coralliens indurés
}

\section{M.P. LUONG \\ P. HABIB}

Laboratoire de Mécanique des Solides,

CNRS UMR 7649 .

École polytechnique, 91128 Palaiseau Cedex
Cet article rapporte une expérimentation en vraie grancleur d'un système d'ancrage LMS à forte capacité de tenue dans un fond marin constitué de calcaires coralliens indurés de la Polynésie française. Les résultats ont mis en évidence la possibilité de reprise des efforts dans des zones où la compacité du terrain exclut la mise en ceuvre des ancres marines traditionnelles. La sécurité de tenue ne dépend pratiquement pas de la couche superficielle souvent hétérogène. La reprise des efforts verticaux réduit très sensiblement la longueur des lignes de mouillage. Le poids réduit de l'ancrage, grâce à une efficacité exceptionnelle ( $>100$ ), est un avantage technique et économique de mise en œuvre, extrềmement appréciable par rapport aux ancres marines de type classique.

\section{Holding performance of LMS anchoring systems in hard coral seafloors}

Anchorage problems are evidently important for the security of vessels, crane barges, with more recent applications in offshore drilling or hydrocarbon production platforms which need high holding power anchoring systems. This paper reports an installation procedure using standard pile driving techniques and full scale testing of a high holding power anchoring system casted in hard coral seafloor areas in French Polynesia. The tested anchoring systems resist loading in any direction, particularly uplift, and offer extremely high holding capacity to size ratios. Where space is limited and drag or clump anchors or stake piles are unsuitable, these small inexpensive anchoring systems can provide very large holding capacities and be installed with readily available marine standard equipment. 


\section{Introduction}

L'intensification des travaux en mer d'une ampleur de plus en plus importante a créé des besoins nouveaux en matière d'ancrage sur fonds marins de nature diverse et variée. L'extrapolation en poids des ancres classiques de marine a permis de faire face à l'augmentation progressive de la taille des supports flottants. Cependant certains cas nécessitent des efforts verticaux importants en point fixe. Ce qui impose un dispositif spécial foncé verticalement dans le sol à l'aide d'un appareil de fonçage traditionnel.

Cet article présente l'expérimentation en vraie grandeur et en milieu marin d'un système d'ancrage spécifíque à forte capacité de tenue, conduite dans un fond constitué de calcaires coralliens indurés.

\section{Description du système d'ancrage}

Le système d'ancrage expérimenté fait partie d'une famille d'ancrages de haute capacité de tenue (Habib et Luong, 1978; Habib et Luong, 1980; Habib et Luong, 1982 ; Habib et al. 1982 ; Habib et Luong, 1987 ; Luong et Habib, 1984). Il se présente sous la forme d'une poutre centrale très robuste sur laquelle sont rapportés (Figs. 1 et 2) :

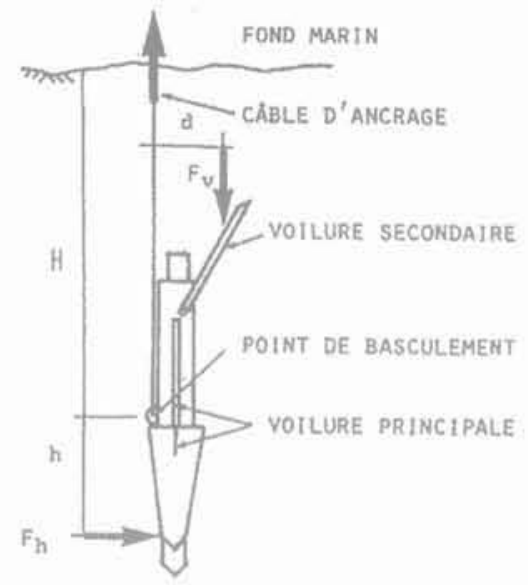

FIG. 1 Description et caractéristiques géométriques du système d'ancrage à forte capacité de tenue testé.

Description and geometrical characteristics of the tested high holding capacity anchoring system.

- A sa partie supérieure :

a) une enclume de battage et un axe assurant le guidage et la jonction avec le faux pieu de battage,

b) une voilure secondaire articulée ou voilure de basculement;

- A sa partie inférieure et médiane :

a) une voilure principale de reprise des efforts de butée dans le terrain ; la partie inférieure de cette voilure peut être constituée par un caisson profilé qui comporte à sa 30 partie basse un outil perforant,

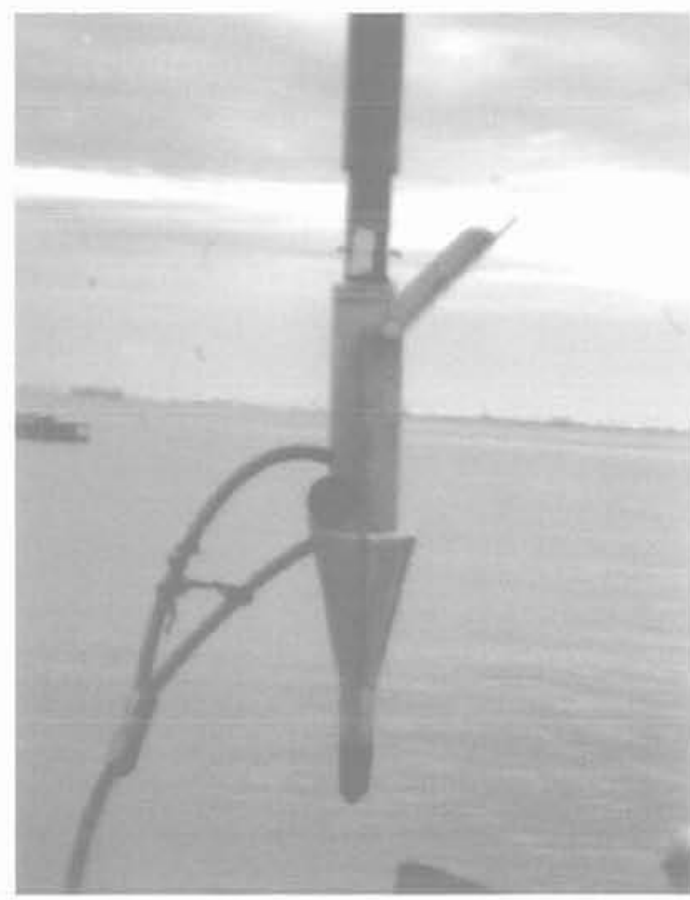

FiG.2 Système d'ancrage à forte capacité de tenue expérimenté en vraie grandeur en fonds marins coralliens.

Full scale experimentation of a high holding capacity anchoring system in coral seafloor.

b) un axe de jonction du câble d'ancrage assure la liaison avec la ligne de mouillage; le basculement de l'ancre se fait autour de cet axe.

\section{3}

\section{Mise en œuvre}

La mise en cuvre se fait par battage à l'aide d'un faux pieu (Fig. 3) qui, après l'enfouissement de l'ancre à la profondeur requise, est déconnecté de celle-ci et récupéré par un marteau vibrant. La voilure secondaire ou voilure de basculement, située sur la partie supérieure de l'ancre, favorise et assure son basculement quand une tension est exercée sur le câble de traction. La tension minimale pour passer de la position verticale de l'ancre à une position inclinée propice à la mobilisation de la butée du sol doit correspondre à une force supérieure au frottement latéral qui s'exerce sur la partie antérieure de l'ancre au contact du sol non remanié.

\section{4.}

\section{Tenue de l'ancrage}

La tenue de l'ancrage est classiquement définie par la force maximale de traction pouvant ètre exercée sur la ligne d'ancrage. En fait, la résistance maximale de l'ancrage est donnée par la tension de la ligne d'ancrage au niveau de son point de fixation avec la structure d'ancrage, qui provoque la rupture du sol au voisinage de l'ancrage. Elle dépend principalement des propriétés mécaniques du sol adjacent, de la profondeur d'enfouissement, de la surface active de l'ancrage et de la rugosité de cette surface (Bemben et al., 1973). 

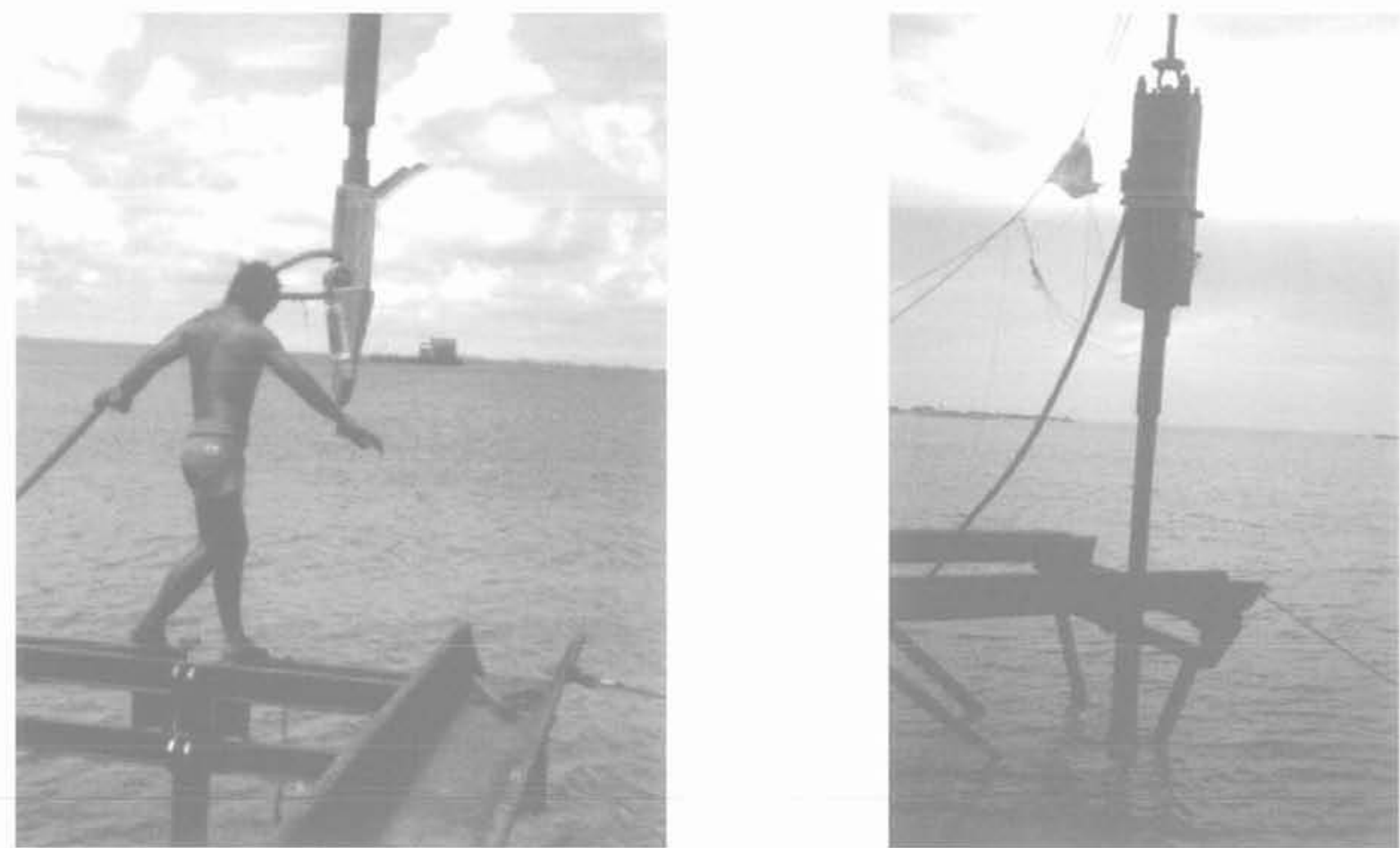

FIG. 3 Mise en œuvre du système d'ancrage à forte capacité de tenue par battage usuel à l'aide d'un faux pieu tubulaire récupérable en fin d'opération.

Installation of the high holding capacity anchoring system using standard pile driving techniques with a follower. recovered after installation.

De très nombreux essais en laboratoire et en place ont mis en évidence deux principaux mécanismes de rupture caractérisant le volume de sol mis à contribution :

- mobilisation de la butée du sol par un mécanisme souterrain conférant un ancrage stable ;

- mobilisation d'un cône d'influence par un mécanisme superficiel pouvant conduire à un ancrage instable.

L'expérience montre que l'effet du mécanisme souterrain se manifeste pour des profondeurs d'enfouissement de l'ordre de quatre ou cinq fois la longueur totale de l'ancre. La tenue d'un ancrage est une fonction rapidement croissante de la profondeur d'enfouissement et peut très largement masquer le rôle des autres paramètres. Les tenues d'un ancrage enfoui dans un massif pulvérulent (sables, graviers) $I_{p}$ et dans un sol argileux $T_{\text {a }}$ sont respectivement données en première approximation par les expressions :

$$
\begin{aligned}
& T_{p}=\gamma \cdot H \cdot S \cdot A_{p} \\
& T_{n}=c . S \cdot A_{n}
\end{aligned}
$$

où $\gamma$ est le poids volumique déjaugé du sol ;

H la profondeur d'enfouissement définie comme étant la profondeur sous la surface initiale du sol du barycentre $\mathrm{G}$ des efforts appliqués à l'ancre (la position du barycentre est fonction du type de sol d'ancrage)

S la surface de la voilure efficace (dont la valeur peut être fonction du type de terrain d'ancrage);

$A_{p}$ un coefficient d'ancrage adimensionnel fonction des caractéristiques mécaniques du sol pulvérulent (frottement, compacité...) et de la profondeur relative d'enfouissement; ce coefficient d'ancrage est déterminé expérimentalement à partir d'essais dans différents types de sols sableux ou graveleux avec des ancres de géométries variées ;

$A_{a}$ un coefficient d'ancrage adimensionnel fonction des caractéristiques mécaniques du sol argileux (compacité, surconsolidation...) ; ce coefficient d'ancrage est déterminé expérimentalement à partir d'essais dans différents types de sols argileux avec des ancres de géométries diverses et variées.

\section{5}

\section{Tests d'ancrage}

Pour évaluer la performance mécanique de l'ancrage, des essais de traction verticale ont été conduits à partir d'une gabarre portuaire correctement ancrée sur l'arrière (Fig. 4). La traction verticale sur le système d'ancrage installé est assurée par virage au treuil à vitesse constante et est mesurée par un capteur de force sur la ligne de traction.

L'instrumentation a été assurée par un dynanomètre en ligne dans la partie située sur le pont. L'enregistrement continu du signal donne la tension sur l'ancrage malgré les mouvements irréguliers du navire dans la houle. Les résultats obtenus (tableau I) lors de ces essais de traction verticale dans un sol induré correspondant à des efforts évidemment bien supérieurs à ceux prévus par la similitude avec les essais effectués en laboratoire dans un sable fin homogène et sans cohésion. Les essais de traction ont été faits deux fois à 


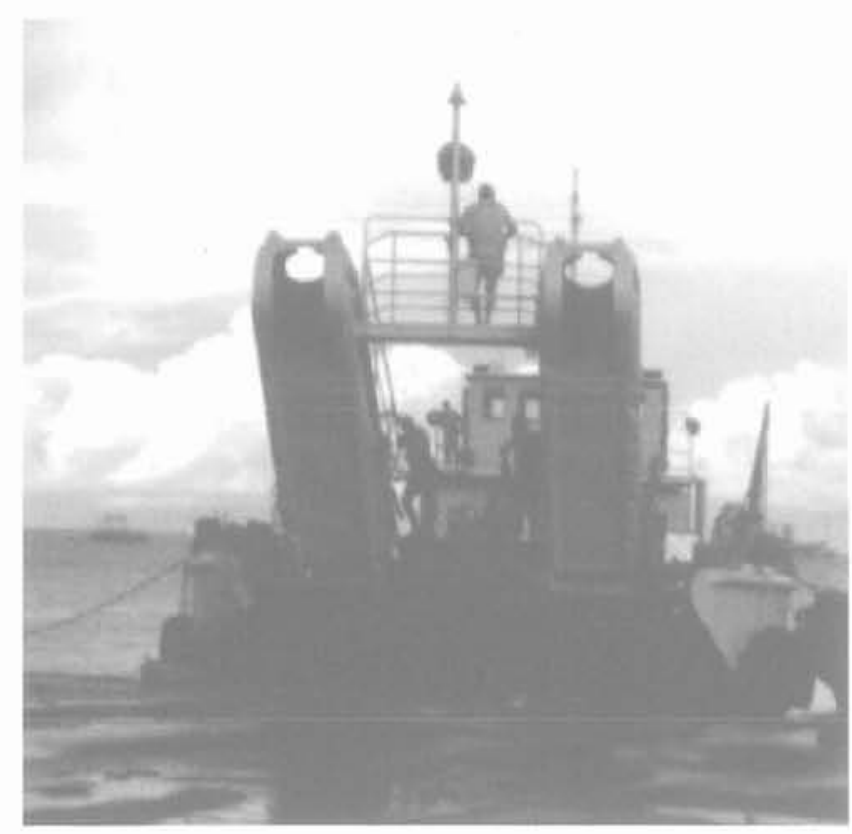

FIG.4 Évaluation en vraie grandeur de la performance mécanique d'un système d'ancrage à forte capacité de tenue.

Full scale proof experimentation on high holding capacity anchoring system.

3 mois d'intervalle. Des forces plus grandes auraient cassé les orins.

\section{6}

\section{Conclusion}

Les performances de ce système d'ancrage à forte capacité de tenue sont remarquables. Les tests de performance mécanique en vraie grandeur réalisés sur site ont permis de mettre en évidence que :

a) la reprise des efforts est possible dans des zones où la compacité du terrain exclut la mise en ceuvre d'ancres marines traditionnelles;

b) la sécurité de tenue est pratiquement indépendance de la couche superficielle qui, très souvent, est hétérogène; c) la possibilité de reprise des composantes verticales d'efforts permet de réduire la longueur des lignes de mouillage :

d) le poids réduit de l'ancrage lui confère un excellent coefficient d'efficacité : une tenue verticale de $390 \mathrm{kN}$ (limite respectée pour éviter la rupture de l'orin) pour un poids de l'ancre de $800 \mathrm{~N}$ correspond à une efficacité de 487 . Ces résultats ont été confirmés par des essais similaires effectués par des chercheurs de la marine américaine avec des systèmes d'ancrage de nature équivalente (Bowman et al., 1994).

\section{Bibliographie}

Bemben S.M., Kalajtan E.H, Kupperman M. - The holding capacity of marine anchors in sand and clay subject to static and cyclic loading, 5th Offshore Technology Conference, Houston, Texas, vol. 2.1973, p. 871-880

Bowman L.E., Forest J., Taylor R.J., Seelig B, - Pile-driven plate anchors for fleet moorings, 26th Offshore Technology Conference, Houston, Texas, vol. 1, 1994, p. 225-232.

Habib P, Luong M.P. - Nouveau systeme d'ancrage. Brevet IFP, BF, n EN 78.13299, 1978.

Habib P., Luong M.P. - Nouveau procédé d'ancrage et dispositifs pour sa mise en œuvre: Brevet ARMINES, BF n 880.17491, 1980.

Habib P., Luong M.P. - Anchoring system. Brevet IFP, United States Patent $n^{\circ} 4,346,663,1982$.

Habib P., Luong M.P. - Articulate element anchorage device having a cranted shape. Brevet IFP-ARMINES, United States Patent $n^{\circ} 4,688,360,1987$.

Habib P., Luong M.P. - Fondations ancrées précontraintes pour pylônes électriques - Prestressed anchored foundations for electric pylons. 12th International Conference Soil Mechanics and Foundation Engineering, Rio de Janeiro, Brasil, August, Balkema, vol. 5, 1989, p. 2991-2992.

Habib P, Le Tirant P., Luong M.P. - $\ll$ Hanchors and model tests of high-capacity anchorings, Geotechnics in a marine environment. o Bulletin technique du Bureau Veritas, English issue, January, vol. $11, n^{\circ} 1,1982$, p. $5-15$.

Habib P., Luong M.P. Martin A. - Ancrages marins en sols coralliens indurés - Marine anchors in indured coral seaffoors. 12th International Conference Soil Mechanics and Foundation Engineerig, Rio de Janeiro, Brasil. August, Balkerna, vol. 2, 1989, p. $1017-1018$.

Luong M.P. Habib P. - Dispositif d'ancrage à élément articulé ayant une forme coudée. Brevet IFP-ARMINES, BF n84.07281, 1984.

TABLEAU I Capacité d'ancrage testée verticalement. Anchoring capacity proof tested vertically.

\begin{tabular}{l|c|c|c}
\hline Types diancrage & $\begin{array}{c}\text { Tenue diancrage } \\
\text { (charge de service) } \\
\mathrm{kN}\end{array}$ & $\begin{array}{c}\text { Force maximale du test } \\
\text { sans rupture 3 mois apres } \\
\mathrm{kN}\end{array}$ & $\begin{array}{c}\text { Enfouissement } \\
\mathrm{m}\end{array}$ \\
\hline LMS 4/2 & $>60$ & 114 & 3,00 \\
\hline LMS 4/2 & $>60$ & 114 & 3,00 \\
\hline LMS 4/2 & $>60$ & 94 & 4,30 \\
\hline LMS 4/2 & $>60$ & 98 & 4,50 \\
\hline LMS 4/2 & $>60$ & 96 & 6,20 \\
\hline LMS 4/3 & $>200$ & 390 & 6,00 \\
\hline
\end{tabular}

